\title{
How far we have come in detecting Circulating Tumor Cells?
}

\author{
Rupam Ghosh \\ Department of Biological Sciences, Indian Institute of Science Education and Research, Indore By Pass Road, \\ Bhauri, Bhopal, Madhya Pradesh, 462066, India.
}

\begin{abstract}
Fluid biopsies dependent upon recognition and profiling of circulating tumor cells (CTCs) offer a non-obtrusive instrument, encouraging unanticipated disease analysis and treatment monitoring. CTCs attack the remote organs by means of the circulatory framework. It has been assessed that primary tumor tosses a million cells day by day into dissemination. Notwithstanding, the majority of these cells bite the dust in the circulatory framework and just the fittest achieve the ordained area to cause secondary tumors. The identification of CTCs in the blood and their characterization can furnish knowledge into the metastatic potential of the primary tumor and the phase of the metastasis. This review aims to provide the reader a glimpse of CTCs, their importance in tumor recognition, different systems for distinguishing and separation of CTCs from blood, their characterization and the challenges ahead to make CTCs a dependable and effective diagnostic device. Such advancement will possibly exchange traditional biopsy presenting new period of customised cancer management.

Keywords: circulating tumor cells(CTCS), cancer stem cells(CSCs), tumor heterogeneity, human epithelial growth receptor 2 (HER2), estrogen receptor alpha(ERo), circulating tumor materials (CTMat), aptamers, AuNP (silver nanoparticles), PCR (polymerase chain reaction
\end{abstract}

\section{Introduction}

Tumor is the one of the heading reason for passings on the planet and the toll of human lives is relied upon to cross 13 million by 2030 [Ferlay et al., 2008, Globocan]. In its preparatory stage, disease might be acknowledged to be a limited illness. However as the stage propels, the sickness spreads to different districts of the form by a methodology regarded as metastasis and it is because of metastasis that the majority of deaths happen in malignancy. The normal 5- year survival rate for patients with confined and metastatic prostate growth is $100 \%$ and $28 \%$ separately (American Cancer Society, 2013). Grasping and concentrating on the purpose behind the metastasis has long been a focal topic in the battle against the debilitating malady. Disregarding the enormous expositive expression on metastatic course, there are numerous unknowns all the while (Jemal, Bray et al. 2011).

Carcinogenesis is the methodology of establishment of tumor cells from ordinary cells. If the typical cells are triggered by inner components or outside variables to turn into a malignant one, is still a matter of debate. Carcinogenesis might be recognized as a Darwinian procedure, comprising of grouping of occasions that could be compressed into four fundamental classifications: essential tumor shaping and development, epithelial to mesenchymal transition (EMT) and intravasation, haematogenous spread of malignancy and extravasation and framing of optional tumor. Haematogenous tissue scattering is the key venture to metastases.

In the course of the most recent decade, biomarkers have been utilized to recognize and stratify patients who might most profit from novel helpful operators. The sign of such exactness therapeutic consideration is the distinguishing proof and remedial focusing of BCR-Abl (break point cluster region gene) with imatinib in patients with perpetual myelogenous leukaemia (CML). Recent triumphs incorporate focusing on Her2 in breast cancer, BRAF gene changes in melanoma3 and EML4 and anaplastic lymphoma kinase (ALK) oncogenic combination genes in non-small unit lung carcinoma(King, Casavant et al. 2014). Numerous creators propose that an underlying many-sided quality exists in progressive strong tumors and metastatic sores that offer ascent to safe clones that escape these therapies. Practicing clinicians are regularly left to depend on tumor aspects (e.g. malady stage),basic imaging modalities (e.g. CT or skeletal substance filters), blood biomarkers (e.g. emitted proteins, for example, CEA or PSA) that may not correspond with either medication reaction or survival and singular patient attributes that anticipate inadmissible poisonous quality from hostile to tumor therapies(i.e. patients with poor renal capacity ought not get chemotherapies that are renally discharged). The most clinically significant growth biomarkers to date remain tissue biopsies. Biopsy tumor specimens experience morphologic, histologic and all the more as of late, genomic aspects to figure out the growth sort and ID of prescient markers for medication reaction. Notwithstanding, investigation of documented tumor biopsies regularly don't reflect the dynamic adjustments that tumors display at the present time metastases. Later cases studies infer that biopsies for thorough examinations can have long-lasting effects on patients. High require in tumor biopsies hinging upon the many-sided quality of the infection is further adding to the worries. The contemporary tumor administration tools, which essential incorporate radiological imaging and serum tumor markers, are lacking in 
tending to the clinical requirement for compelling clinical disease administration. To enhance the progression free survival (PFS) and overall survival (OS) of disease patients, novel and profoundly powerful biomarkers that uncover the molecular cosmetics of the sickness are direly required. Such biomarkers will enable personalized therapy,therapeutic efficacy monitoring, and potentially early diagnosis of cancer.

The presence of CTCs in cancer patients was first detected in 1869 by an Austrian physician Thomas Ashworth who discovered that the blood samples of cancer patients contain small cells which resemble the cells of primary tumor (Ashworth, Med. J. Aust. 1869). The belief that CTCs may act as surrogate source of tumor cells triggered intensive studies in this field. The enumeration of CTCs with the survivability of cancer patients confirmed the role of CTCs in cancer progression (Fehm, Sagalowsky et al. 2002, Fidler 2003).

Once the CTCs are shed by the primary tumor, they travel to distant organs of the body not only by the lymphatic system by also through blood and form secondary tumors(Chambers, Groom et al. 2002).CTCs have been identified in significant numbers in patients with metastases but not in healthy or people with benign Tumors.

But all the CTCs do not have the potential to give rise to secondary tumors (85\%) and perish within 2.5 hours. Inside the circulatory system, they are subjected to different fates. Studies by Dunn et al. showed that they undergo anokis, disintegration by immunoediting or transition to dormant stage while remaining in the circulation. They can also undergo cell death following extravasation or they can remain dormant for a long period of time (5-25 years) without subsequent aggressive proliferation. If a CTC successfully evades all the above fates in the circulatory system, it can develop a malignant macro metastasis. Although they are extremely rare in the circulatory system, their spread is a necessary condition for metastasis(Hanahan and Weinberg 2000, Cristofanilli, Budd et al. 2004, Hayes and Paoletti 2013). The diameters of CTCs are 3-4 times as large as the pores of the blood capillaries. This means that only a small amount if CTCs remain in circulation, which can be detected. Also, these CTCs lodge in the capillaries and undergo apoptosis.

Recent studies have revealed that CTCs express both stem cell markers and mesenchymal traits, indicating the possibility to transitorily acquire stem cell competence following the passage through epithelial to mesenchymal transition (EMT). CTCs can be subdivided into three sub-populations: cancer stem cells, tumor amplifying cells and tumor initiating cells arising from epithelial cancer cells of the primary tumor which had undergone EMT(Yu, $\mathrm{Ng}$ et al. 2013). EMT can also induce non-CSCs to enter a CSC like state generating CSCs de-novo. The connection between CTCs and CSCs is still unclear(Gupta, Chaffer et al. 2009). Also, CTCs that are not of melanoma origin have low expression of epithelial markers.

The role of circulating tumormicro emboli (CTM) in metastatic development has come up in the recent years. CTM consists mainly of tumor cells (at least 2) and blood cells. CTMs have been observed in peripheral blood of metastatic lung,liver, colorectal, renal, breast and prostate (Brandt, Junker et al. 1996, Molnar, Ladanyi et al. 2001, Witzig, Bossy et al. 2002, Vona, Estepa et al. 2004, Paterlini-Brechot and Benali 2007, Hou, Krebs et al. 2010, Stott, Hsu et al. 2010, Hou, Krebs et al. 2011). The morphology of CTM is highly variable and form as a result of the increasing adhesive character of CTCs. Increased study suggests that the presence of CTM could be an indication of higher metastatic potential $[22,23,25,27]$. The conceivable illustration for the expanded metastatic potential of CTM, when contrasted with CTCs is because of: i) CTMs are more trapped in the tight vasculature than CTCs and ii) CTMs present a favourable microenvironment for tumor cell survival. Likewise, extravasation is not an essential for CTM mediated metastasis.

Comprehending the aspects of CTCs is clinically critical for the advancement of unanticipated tumor intercession procedures and medicines.

\section{CTC isolation and enrichment}

Over the last decade, a number of strategies to isolate CTCs from billions of RBCs and leukocytes have been performed. Two factors make the detection and separation of CTCs extremely challenging: i) CTCs are extremely are and occur at the rates as low as 1 in $10^{9}$ erythrocytes and 1 in $10^{7}$ leukocytes ii) there is no one marker to detect CTCs specifically. Leukocytes share many properties with CTCs leading to high level of leukocyte contamination in many enriched samples( $\mathrm{Yu}, \mathrm{Ng}$ et al. 2013).

Due to the extremely low number of CTCs in blood circulation, an initial enrichment step is done to separate the majority of blood cells and from the sample. CTC enrichment is done using various methods like microfluidic CTC chips, magnetic particles, microfiltration, dielectric separation and flow cytometry. Once enriched in a particular region (most preferably beneath the skin), CTCs are easy to isolate.

As CTC counts are associated with patient prognosis, a highly sensitive detection strategy is vital to accurately identify and enumerate the CTCs isolated. The performance of a typical CTC detection system is evaluated on the basis of the parameters as given in the Table1.

The CTC detection techniques are based on mainly two factors - physical properties (like size, density, electric charge and deformability) and biological properties (like protein expression and invasion capacity). 


\subsection{Physical properties based methods}

The difference in biophysical and biomechanical properties of CTC and leukocytes has been used for CTC separation since many years(Jin, McFaul et al. 2014). In the early 50s with the wide spread use of Papanicolaou stain, CTCs could be identified with greater certainty. Physical features like lobular, crenelated, elongated nuclei and fragmented chromatin became the signs of malignancy. Later studies found that CTCs have many properties that could be used for differentiating CTCs form blood cells. Some of these properties include specific gravity, size and rigidity. Cluster forming ability of CTC can also be harnessed for label free isolation techniques.

Automated microscopy and picture investigation of substantial number of fluorescently tagged cells have shown the normal diameter of CTCs to associate with 7-9 $\mu \mathrm{m}$ as contrasted with 6-9 $\mu \mathrm{m}$ of leukocytes. Because of noteworthy overlapping of sizes, leukocytes may thwart the division routines. Contrasts in nuclear to cytoplasmic proportion (N/C ratio) of CTCs and leukocytes exceed by an order of magnitude or more. N/C ratio determines deformability of the cells which might be utilized to as an alternate critical property in CTC partition. Shape, thickness, electrical polarizability and magnetic susceptibility could be utilized for disengaging CTCs from blood.

Size based isolation has been acknowledged utilizing track carved polycarbonate channels, which are likewise utilized in the business ISET and ScreenCell framework for the seclusion of CTCs. The channel is a permeable layer holding various haphazardly disseminated $8 \mu \mathrm{m}$ measurement openings that permit blood constituents to cross but catch the bigger CTCs(Esmaeilsabzali, Beischlag et al. 2013). Cells frequently remain sound after the separation process, allowing their ensuing morphological or molecular investigation. Size based disconnection could be performed in the microfluidic setting where the reconciliation of exactly characterized topology of microstructures with the laminar stream in the microchannels offer novel answers for cell detachment. Filtration is the methodology of streaming a specimen through a show of micro-scale choking influences with a specific end goal to catch target cells dependent upon the blending of size and deformability. Filtration microstructures could be classified into three essential sorts - weir, column and pore. Weir structures comprise of micro channels holding a sudden abatement in the channel cross area. Column structures comprise of an exhibit of micro posts dispersed suitably to structure choking influences to catch target units. The pore structure comprise of membrane punctured with two dimensional clusters of little gaps. The key outline parameters in these channel microstructures incorporate the stream rate of liquid and the cross sectional opening of the constriction. The liquid stream rate verifies the force connected to each one cell as it is distorted through the constriction. The cross sectional opening figures out the size and state of the cell that might be caught by the channel. Utilizing this rule, around $85 \%$ of cells could be caught as depicted by Lin and group (Alix-Panabieres and Pantel 2014). Be that as it may the key test in utilizing mechanical stipulation to differentiate these cells is the potential of clogging when vast amounts of cells are transformed, which can cause eccentric varieties in the stream rate and therefore in the force connected to squeeze the units through every narrowing. Other challenge is the extraction of cells as they get adsorbed onto the channel material effectively.Use of crescent shaped traps help to prevent clogging by accumulation of cells and other debris.

Hydrodynamic chromatography utilizes associations between particles and hindrances in stream so as to confer diverse stream speeds to distinctive particles dependent upon size contrasts and deformability (Jin, McFaul et al. 2014). Hydrodynamic chromatography has been utilized within two separate ways. The first works in the low Reynolds number administration where inertial impacts might be overlooked while the second one works with the Reynolds number of the order of one, where the inertial impacts couldn't be disregarded. Lateral displacement of cells could be utilized as a vital property within the low Reynolds number administration. Hydrodynamic methodologies can achieve higher throughput than filtration strategies, however the enrichment is regularly poorer which is because of its powerlessness to separate nucleated cells, dependent upon deformability.

Dielectrophoresis (DEP) is fit for segregating between blood cells and tumor cells dependent upon the properties of unit size, nuclear and membrane morphology(Jin, McFaul et al. 2014). The point when a cell is suspended in a dielectric medium distinct from the cell and is subjected to an electric field, a dipole moment along the course of the connected field is created because of charges affected at the interfaces. In the event that electric polarizability of the cell surpasses that of the suspending medium, the DEP power will be along the same course as the slope of electric field. Hence, cells move towards the solid electric field locale, bringing about positive dielectrophoresis. Then again, when the electrical polarizability of the units is less than that of the medium, the cells move towards less electric field district. Yield up to $95 \%$ could be attained by this procedure. Contrasted with filtration and hydrodynamic strategies, dielectrophoresis routines as of now slack in execution in both selectivity and throughput.

Microfluidic devices combining multi-orifice flow fractionation (MOFF) with dielectrophoresis (DEP) and dielectrophoretic field flow fractionation (DEP-FFF) are also gaining space in the field due to combinatorial approach of different size and membrane properties(Alix-Panabieres and Pantel 2014). 
Cells might be recognized as dielectric particles because of the polarity and the conductivity of the cell membrane and cytoplasm(Esmaeilsabzali, Beischlag et al. 2013). The cell when placed in the influence of spatially inhomogeneous AC field, taking into account dielectric lands of the cell, the convey medium, the recurrence and the adequacy of the field, they will encounter distinctive electrokinetic strengths. These electrokinetic might be utilized for characterization and seclusion CTCs. The only drawback in this process is relatively slow processing rate, which limits the rate of sample processing.

Density gradient centrifugation has also been used to separate CTCs but a significant amount of CTCs were lost in this case and hence the method was not encouraged further, though some systems like OncoQuick are based on this principle(Arya, Lim et al. 2013, Alix-Panabieres and Pantel 2014).

Variability in the size of cancer cells of different types and within different cancer cell lines combined with the lack of standard definition of performance metrics are the main drawbacks of these physical properties bases separation techniques. Training the device to tackle different types of cancer cell line as well as adopting standard definition of performance cut-offs can mitigate the problem.

\subsection{Biological properties based methods}

Nucleic acid based methods catch particular DNA or mRNA particles in the example to distinguish the presence of CTCs(Phillips, Xu et al. 2009, Xu, Phillips et al. 2009, Cerchia and de Franciscis 2010). These genes code for tissue, organ, and tumor particular polypeptides with known mutations found in cancer cells. This methodology is most delicate consistent with an exactness of 1 CTC in 5-6 million cells processed. CTC recognition utilizing mRNA molecules are by and large more successful. Anyhow nucleic acid based system has certain disadvantages- CTCs must be lysed before PCR process which avoids their immediate observation, identification and post separation examination. Further, the specificity of the process reduces the overall accuracy of the approach as truly CTC specific biomarker is yet to be discovered. So, false positives are also detected which include the small number of non-cancer cells that have entered the circulatory system due to inflammation, diagnostics biopsies and tumor surgeries.

As CTCs are shed from solid tumors daily and the majority of them getdestroyed, the intracellular components like DNA, chromatin, proteins remain in the circulatory systemforming circulating tumormaterials (CTMat). CTMat can also prove to be very useful diagnostic tool as they are present in more number than CTCs(Hong and Zu 2013).

Aptamers (single stranded RNA and DNA) have also demonstrated potential as an alternative identification method(Xu, Phillips et al. 2009). The molecular weight of aptamers is around $15 \mathrm{kDa}$ and they are highly resistant to adverse conditions like $\mathrm{pH}$, temperature and chemicals. The technique of Systematic Evolution of Ligands by Exponential Enrichment (SELEX) has been employed to facilitate the automation of in vitro aptamer production. When compared to antibodies, the manufacture of aptamers is much easier and faster. It has been suggested that one aptamer binds to one specific antigen on the rare cell. The aptamers can distinguish CTCs from normal cells more precisely. An aptamer based, cell affinity chromatography micro device has been developed that is capable of isolating tumor cells from a mixture of control cells and sort them. The enrichment factor of the micro device is determined to be around 130 times.

Telomerase are known to be actuated in majority of malignancy of disease sorts and is known to be connected with metastatic properties(Hong and $\mathrm{Zu}$ 2013). The movement of telomerase have been found in blood samples of cancer patients. Since, lifted level of telomerase is one of the signs for stem cell characteristics; this could be utilized to distinguish CSCs also. CTCs might be productively identified by telomerase based action however entire blood cells must be lysed first which might thwart endeavours to do catch up investigation of CTCs.

Antigen antibody based bindings are always more efficient and effective approach for detection and isolation of CTCs(Alix-Panabieres and Pantel 2014). Antibody based CTC isolation is done using an antibody based matrix to which CTCs are specifically bound and either directly or indirectly captured. CellSearch System is the most widely used assay to detect CTCs. It is based on an anti-EpCAM immunomagnetic capture step followed by fluorescent images that capture nucleated (DAPI +ve), cytokeratin +ve, CD45 -ve events that are designated as CTCs. Other CTC detection technologies based on EpCAM include a microfluidic chip (CTCChip) which contains EpCAM antibodies on the chip platform, HB- Chip (herringbone chip), Ephesia which uses columns of bio functionalized super paramagnetic beads assembled on a microfluidic channel onto an array of magnetic straps, Magsweeper which can also perform post separation analysis and Velcro like microfluidic device. Besides antibody binding, other approaches like E-Selectin and Peptide binding have also been studied. Immunomagnetic systems have been used to identify CTCs. CTCs are initially tagged with antibody conjugated attractive microbodies or nanoparticles that frequently bind to a particular surface antigen or intracellular antigen. The cells when laid open to a non-uniform magnetic field, the labelled cells relocate contrastingly and are caught. The execution of an immunomagnetic technique is dependent upon expression and specificity of 
target antigen and binding viability of the antibody, effectiveness of immunolabelling methodology and level of detachment in the attractive field.

Microfluidic bead based nucleic acid sensor can selectively isolate CTC from blood sample(Esmaeilsabzali, Beischlag et al. 2013). In this method, micro beads functionalised with HRP and DNA probes act as labels. So, two signal amplification approaches are integrated for enhanced detection of CTCs.

The basic blemishes connected with contemporary biochemical based methodologies is the epithelial to mesenchymal transition (EMT) of tumor cells which prompts the loss of epithelial markers on which dominant part of immunological strategies are based. Additionally, the detachment procedure from blood causes perpetual connection of the cells to the surface and subsequently restrains their extraction and characterization.

\subsection{Combination Assays}

Combining physical and biochemical properties of tumor cells can increase the efficiency and accuracy of detection procedures. A combination of size based hydrodynamic cell sorting and immunomagnetic selection has been designed into a device CTC-iChip by Ozkumar et al.successfully.

Modifying topological properties along with biochemical ones can enhance cell adhesion upto 10 folds as the local interactions between nano structured surface and cell membrane are increased(Esmaeilsabzali, Beischlag et al. 2013). This forms the basis of adhesion based methods of CTC isolation. Studies have indicated that this method captures more invasive subpopulations of CTCs and the cell also remain viable after separation procedure.

Asghar et al., portrayed the creation of strong state micropores in silicon for electrical finger printing, $3 \mathrm{D}$ profiling and discovery of tumor cells. Under enhanced states of stream rate, they were fit to separate tumor units from blood cells, attaining a CTC recognition effectiveness of $70 \%$.

The Nanovelcro CTC Chip, a unit made out of a designed silicon nanowire substrate (Sinw) and an overlaid polydimethylsiloxane (PDMS) confused blender. Accepted by two organizations partaking in the study, the Nanovelcro Chip result displays extremely predictable effectiveness in CTC-isolation from patient samples( $\mathrm{Lu}$, Zhao et al. 2013).

Only $50 \%$ of patients with metastatic breast cancer have elevated CTC levels and even if the levels are high, their relative number in the total blood volume is very lesswhich limits the efficiency of the detection process. An assay that increases the sensitivity is more prone to capture non-malignant cells emphasizing the importance of second characterization step in the assay. Also, there is a possibility that a captured cell which is morphologically and genetically identical to a cancer cell may not have the same metastatic potential. Presence of microscopic malignant cells at sites external to the primary tumor is associated with poor prognosis. The presences of malignant cells do not imply absolute risk of subsequent recurrence. Meng et al. reported that $36 \%$ women who had no evidence of clinical disease 7-22 years after mastectomy has detectable aneusonic CTCs. Reports by Weidswang indicated that $15 \%$ who were diseases free after 3 years of follow up had bone marrow micro metastases. After a subsequent follow up period of approximately 3 years, only $21 \%$ of these patients with documented persistent bone marrow metastases relapsed. Overall, these studies show that high sensitive assays may not have clinical implications as predicted. But molecular characterization of CTC will play an important role in relation to targeted therapy.

\section{CTC characterization}

When CTCs are enhanced and gathered; characterization techniques are required to further research their starting point and hereditary profile. Some components must be contemplated while selecting a suitable system for CTC identification(Hong and $\mathrm{Zu} 2013$ ).

\subsection{Immunological detection}

Keeping in mind the end goal to recognize just reasonable CTCs, the practical Epithelial Immunospot (EPISPOT) test, joined with any sort of improvement step, was presented for CTC breakdowns. Maintaining a strategic distance from immediate contact with the target cells, this strategy surveys the vicinity of CTCs dependent upon secreted, shed or discharged proteins. EPISPOT has been connected to blood and skeletal substance marrow (BM) samples of bosom, colon and prostate growth patients furnishing first clinical data. Furthermore, ultra rapid mechanized computerized microscopy utilizing fiber-optic-array scanning technology has been created to catch CTCs that are labelled by antibodies with fluorescent conjugates. Immunological detection offers the focal point of consequent seclusion of stained CTCs for ensuing molecular characterization.

\subsection{Molecular detection}

Tests focusing on particular mRNAs are the most broadly utilized alternate choices to immunological examine to recognize CTCs. Numerous genes are expressed at low level in normal cells, however, expand their expression throughout malignancy. Quantifying their expression level with RT-PCR can set the cut-off for 
precise characterization of samples. The identification of tumor-particular DNA changes is the most particular methodology to locate CTCs; notwithstanding, the checked hereditary heterogeneity of robust tumors represents an issue on the grounds that distinctive tumors of the same element (e.g., breast carcinomas) and even units inside a singular tumor harbour diverse hereditary aberrations. Moreover, hereditary dissection of the essential tumor may not foresee the DNA variations on CTCs and seclusion and immediate hereditary examination of single CTCs are actually extremely testing.

\subsection{Nanotechnology based detections}

Nano oncology is the most important chapter of nano medicine that has redefined detection and diagnosis of cancer(Jain 2010). Gold nanoparticles conjugated with tumor specific antibody target CTC and generate fast electrochemical response that is detected with electrochemical methods. Quantum dots conjugated with tumor specific antibodies that bind to tumor specific antigens can also detect CTC specifically that can be detected by MRI and fluorescence microscopy. Quantum dots can also bind to methylated DNA which can then be lighted up and identified by spectrophotometer.

\subsection{Electrochemical Techniques}

As of late, electrochemical strategies, for example, electrochemical impedance spectroscopy (EIS) and voltammetric procedures have picked up premium in the identification and quantification of CTCs in pre enriched samples(Jin, McFaul et al. 2014). These methods have been utilized for CTC discovery with up to single cell resolution. Numerous studies by research all over the world have produced numerous remarkable results; however the most wonderful ones are by Choi et al. who depicted the label free CTC counting utilizing DC impedance based microcytometer by exploiting the distinction between the span of CTC and blood cells. For estimation they manufactured a microfluidic gadget with two polyelectrolytic gel cathodes and caught change in DC impedance relating to the volume of the cell between terminals under low DC voltage. With proposed gadget they were able to recognize CTCs in 24 out of 24 BC patient specimens. Subsequently, such an apparatus could be utilized for CTC assessment paying little heed to their biomarkers.

\subsection{Photoacoustics based detection}

Photoacoustic imaging has been used for molecular imaging but background signal degrade the detection sensitivity and specificity of targets(Nedosekin, Juratli et al. 2013). Most of the noise is due to optical absorbers which can be removed if magnetic nanoparticles are used for imaging. Magnetomotive photoacoustic imaging helps in the visualization of signal generating objects to great accuracy. Hidden micro metastases can be detected using immunomagnetic assays with magnetic nanoparticles that is made up of carbon coated pure iron core coated with EpCAM antibodies. Photoacoustic flowmetry, making utilization of the broadband assimilation range of melanin, has been tried to identify melanoma cells and has been joined together with nanoparticles focusing on unit surface antigens to expand its relevance in CTC detection. Apart from photoacoustics, photothermal detection of nanoparticles conjugated to CTCs have been demonstrated successfully.

\subsection{Chemiluminescence}

Chemiluminescence has been used for the identification of CTCs utilizing AuNPs (gold ( $\mathrm{Yu}, \mathrm{Ng}$ et al. 2013)nanoparticles) as catalysts for the luminol- $\mathrm{H}_{2} \mathrm{O}_{2}$ reaction(Zhang, $\mathrm{Fu}$ et al. 2013). The high surface-area tovolume proportion of AuNPs and its catalytic properties towards $\mathrm{H}_{2} \mathrm{O}_{2}$ diminishment empowered luminol to be oxidized to generate the chemiluminescence indicator, which was identified with a photomultiplier tube (PMT). The roles of AuNPs as a versatile CTC catching component, an impetus and sign intensification component for chemiluminescence highlight how nanotechnology is influencing CTC detection strategies.

\subsection{Electrical detection}

Electrical discovery is an easy, label free technique which can give constant quantitative recognition of CTCs(Yu, Ng et al. 2013). This identification technique could be effortlessly interfaced with size-based affinity, ligand-based affinity and DEP-based CTC capture or partition methods. Changes in impedance or conductivity as CTCs go between a couple of cathodes could be utilized to identify the vicinity of CTCs.

\subsection{Surface Acoustic Waves}

Surface acoustic wave (SAW) is a delicate, label free technique that might be utilized to catch the coupling of CTCs to ligands in real time( $\mathrm{Yu}, \mathrm{Ng}$ et al. 2013). Interdigitated transducers (IDTs) change over an electrical sign into a SAW by utilizing a piezoelectric material and the SAW is transmitted by a managing layer. As the coupling occasion changes the mass and thickness at the sensing interface, changes in stage and abundance of the SAW happen. The SAW is then changed over once again to an electrical indicator utilizing the 
IDTs and caught. A nanostructured SAW microfluidic chip was utilized for the location of CTCs which generated critical effects.

\section{$3.9 \mu N M R$ detection}

Micro-atomic attractive thunder ( $\mu \mathrm{NMR})$ is a developing engineering, which is exceedingly well with the utilization of magnetic NPs functionalised with legends for CTC catch(Castro, Ghazani et al. 2014). As every CTC is bound by numerous ligands functionalised attractive NPs and every NP can likewise tie to some CTCs, they structure dissolvable nanoclusters and reason a diminish in the T2 transverse unwinding time of billions of nearby water atoms, prompting indicator enhancement and the mass change in $\mathrm{T} 2$ transverse unwinding time could be recognized, which are characteristic of the articulation levels of the surface antigens on the cells. This technique offers high detection sensitivities overcoming technical barriers.

\subsection{Other methods}

A few different systems dependent upon visual microscopy, Atomic force microscopy (AFM), micropipette aspiration and optical tweezers are additionally being considered for further enhancement of recognition methods of CTCs. Nanostructures substrates have also emerged as a promising platform for better specificity in CTC detection. However, non-specific binding and detachment of the cell from the nano-substrate remains an issue.

Tumor derived microvesicles have been proposed as biomarkers for blood based cancers(Kim, Yong et al. 2014). Zwitterionic sulfobetaine-conjugated immunobeads are readied utilizing cluster of separation 83 (CD83) as a hopeful protein marker for breast tumor derived microvesicles. The zwitterionic immunobeads are more than 10-fold proficient for separating tumor associated microvesicles (TMVs) from clinical plasma samples by stifling nonspecific protein tying than ordinary immunobeads.

One of the major challenges in the therapies against metastases is tumor heterogeneity. Tumor heterogeneity is observed between different metastatic sites and the original primary tumor and even within a single tumor at metastasis or primary site. Tumor heterogeneity arises mainly from two factors- i) genetic instability which causes fixed 'genetic' changes in cancer cells and ii) differential but plastic expression of various genes which causes resistance to anticancer therapies.

\section{Detecting CTCs in vivo}

CTCs can be targeted directly either by using the Gilupi nanodetector(Alix-Panabieres and Pantel 2014). It also based on anti-EpCAM antibodies for detection. The alternative approach is the use of leukapheresis and subsequent analysis by flow cytometry and real time PCR for molecular characterisation.

Most of the modern techniques in CTC diagnosis are either detection or isolation separately. Nano-technology combined with X-ray radiation can provide an integrated approach to all the three requirements- enrichment, isolation and elimination of CTCs(Hossain, Luo et al. 2012). In this technique, bismuth and magnetic iron oxide nanoparticles coated with folic acid are used to target CTC in vivo as CTC over express folic acid receptors on their surface. Using micromagnets, CTCs can be enriched to a particular region (beneath the skin) and then exposed to X-ray which leads to complete destruction of these cells with minimum toxicity to neighbouring normal cells.

Utilizing hybrid nanoparticles (HNPs) can perform synchronous detection, in situ protein expression analysis, and cell phenotype of circulating tumor cells (CTCs) (Lee, Cho et al. 2013). Each HNP comprises of three parts: (i) antibodies that bind particularly to a known biomarker for CTC, (ii) a quantum dot that radiates fluorescence signs, and (iii) biotinylated DNA that permits isolation and release of CTC-HNP complex based on chip technology.

\section{Post separation analysis}

The vast majority of the separation strategies lead to the loss of CTCs for further investigation. Remaining suitable disengaged CTCs are utilized for downstream investigation to recognize understanding particular drug susceptibilities and configure customized prescriptions. The hereditary dissection and xenograft studies can further disentangle the instruments of tumor progression and tumor resistance. Invitro functional assays of CTCs have led to the discovery of many markers like MUCIN-1(Hong and Zu 2013). Xenograft studies indicate that CTCs have more metastatic potential than parental cell lines indicating their stem cell like properties.Insight into cell signalling pathways like Wnt signalling have likewise been found from these studies. The examination of CTCs emulating separation step will at last figure out the suitability of an isolation and enrichment methodology. 


\section{Drawbacks and Challenges ahead}

Circulating tumorcells (CTCs) represent a potential surrogate for tissue-based cancer and hence to monitor serial in tumor therapies. Important biomarkers including oestrogen receptor alpha and HER2 have been used for a long time in these assays. The failure of cancer therapy is also intrapatient heterogeneity of tumor cells which provides a route for resistance. Mathematical modelling shows that genetic instability would resultin accumulation of mutations and resistance to cancer therapy. Interpretation followed by successful clinical application has been proved as a major hindrance in which mathematical modelling can play an important role(Hong and $\mathrm{Zu}$ 2013). It helps to distinguish rational data from a set of experimental data. Many questions like the lowest number of CTC that can be detected in a given sample of blood, how can this number give an estimate of total CTC count in the blood and also defines the methodological constraints of cell search system and flow cytometry.Skipper $e t$ al. also showed that a cytotoxic effect of chemotherapy works best when the tumor transplants were recently grafted than the transplants that were allowed to grow for a long time. The expression of biomarkers like HER2, ER alpha is different in secondary tumor than from the primary tumor they arose and this is the inherent property of the tumor cells. The expression level these biomarkers vary greatly from no expression level to high levels of expression. So, the adjuvants that are targeted towards these markers (mTOR, ER alpha) and not successful in case of all patients.

Use of same kinds of chemotherapy for different patients with metastatic disease is widespread which causes the tumor to gain resistance for not only one type but all types of chemotherapy. This has led to more and more stress on personalized medicine strategy. For eg. persons treated with serial endocrine therapies develop resistance towards chemotherapy and vice versa

The great hope of CTC research lies in its use to be used as liquid biopsies that would permit more frequent and minimally invasive tumor cell tracking unlike the traditional methods. Separation of CTCs from blood has been done by various methods based on epithelial antigens; electromagnetic properties of cells and blood flow dynamics, nanotechnology based methods and combined strategies. Use of micro post CTC chip has enabled researchers to capture viable CTCs and culture them efficiently. Monitoring the functionality of CTCs has been helpful in drug testing, chemotherapy response and metastasis stage diagnosis. Recently, a combination of epithelial and mesenchymal cell surface markers has been used to capture all CTCs in blood. The role played by platelets in CTC invasion and EMT is coming up now and offers a new dimensions to design strategies based on platelets since they are more in number. The integrated approach to enrich, detect and eliminate CTCs within the body of the patient can be thought on the basis of chemotactic signalling also. Most the metastatic cancer cells are chemotactic to certain factors(Varani 1982). These factors could be specifically injected into a defined region of the body followed by a particular incubation time to attract the CTCs. Once the CTCs are detected in significant numbers, they could be irradiated from outside or other elimination strategies can be adopted to get rid of them.

To improve the early detection and therapy of cancer cells, better and more efficient ways has to be found for the quantitative and qualitative analysis of circulating tumor cells. However, the detection of CTCs is itself a challenging task and the lack of reliable markers to detect these cells specifically has hindered the development in this field. Till date, no CTC platform is approved to guide clinical care. Still a lot of things need to be done to establish the detection and isolation of CTCs to predict the metastases stage in patients. Developing personalized medicine based on CTC is also a hope to cancer patients.

\section{Acknowledgement}

I would like to acknowledge Kishore Vaigyanik Protsahan Yojana (KVPY), Indian Institute of Science for funding me. I would also like to thank Dr. Apurba Lal Koner, Assistant Professor, IISER Bhopal for going through the manuscript and providing necessary guidance.

Tables and Figures

Table 1 Evaluation Parameters of a CTC detection system

\begin{tabular}{|l|l|}
\hline Parameter & Importance \\
\hline Recovery rate & $\begin{array}{l}\text { It gives the ratio of detected CTCs to total number of CTCs present in the sample. This step is crucial } \\
\text { to know the stage and early diagnosis of cancer. }\end{array}$ \\
Enrichment rate & $\begin{array}{l}\text { It is equal to the ratio of detected CTCs to all cells in the sample. It defines the sensitivity of the system } \\
\text { towards CTC isolation. } \\
\text { It gives an account of the ratio of CTCs to blood cells before and after enrichment. It also indicates the } \\
\text { sensitivity of the system for CTC isolation and gives an idea about the specificity of the } \\
\text { marker/property used for the isolation step. } \\
\text { Throughput } \\
\text { Viability }\end{array}$ \\
$\begin{array}{l}\text { It indicates the speed by which the sample is processed. } \\
\text { It indicates the proportion of viable CTCs to all isolated CTCs. }\end{array}$
\end{tabular}


How far we have come in detecting Circulating Tumor Cells?

Table 2 Various CTC isolation and enrichment technologies

\begin{tabular}{|c|c|c|c|c|c|c|}
\hline Method & Principle & Recovery & Purity & Throughput & $\begin{array}{l}\text { Cell } \\
\text { Viability }\end{array}$ & Sensitivity \\
\hline ISET & Size based & High & Medium & 5 minutes & High & High \\
\hline DEP & $\begin{array}{l}\text { Dielectric property } \\
\text { based }\end{array}$ & Variable & Medium & $\begin{array}{l}30 \text { million cells/ } \\
0.5 \text { hours }\end{array}$ & Medium & Medium \\
\hline Inertial force & Size and weight based & Low & Low & $\begin{array}{l}1 \text { million cells/ } \\
\text { min }\end{array}$ & High & Medium \\
\hline Cell Search & $\begin{array}{l}\text { Immunomagnetic and } \\
\text { fluorescence imaging }\end{array}$ & Medium & Low & $\begin{array}{l}7.5 \mathrm{~mL} \text { blood} / 90 \\
\text { minutes } \\
1\end{array}$ & Not viable & High \\
\hline Magsweeper & Immunomagnetic based & Medium & Medium-High & $\begin{array}{l}20 \quad \text { minutes/ } \\
\text { detection round }\end{array}$ & High & High \\
\hline Micropore Chip & Size and elasticity & Medium & Low & $\begin{array}{l}0.1 \\
\text { blood/hour }\end{array}$ & Low & High \\
\hline CTC iChip & $\begin{array}{l}\text { Combination of physical } \\
\text { and biochemical } \\
\text { properties }\end{array}$ & $\begin{array}{l}\text { Medium- } \\
\text { High }\end{array}$ & Low & $\begin{array}{l}10 \quad \text { million } \\
\text { cells/sec }\end{array}$ & N/A & N/A \\
\hline $\begin{array}{l}\text { Nanostructured } \\
\text { substrates }\end{array}$ & $\begin{array}{l}\text { Bio affinity and surface } \\
\text { topology based }\end{array}$ & High & N/A & Within 30 minutes & High & High \\
\hline
\end{tabular}

Table 3 Post separation analysis

\begin{tabular}{|l|l|}
\hline Methods & Inferences \\
\hline Size and Morphology & Physical characteristics \\
Deformability assays & Physical characteristics \\
ELISA, PCR, Transcriptome analysis & Biomarker characterisation \\
DNA sequencing & Genetic aberration \\
FISH and Immunofluorescence & Enumeration, detection and identification \\
Functional Assays & Drug efficiency testing and cell signalling processes \\
Xenograft Studies & Biochemical pathways and Stem cell properties \\
\hline
\end{tabular}

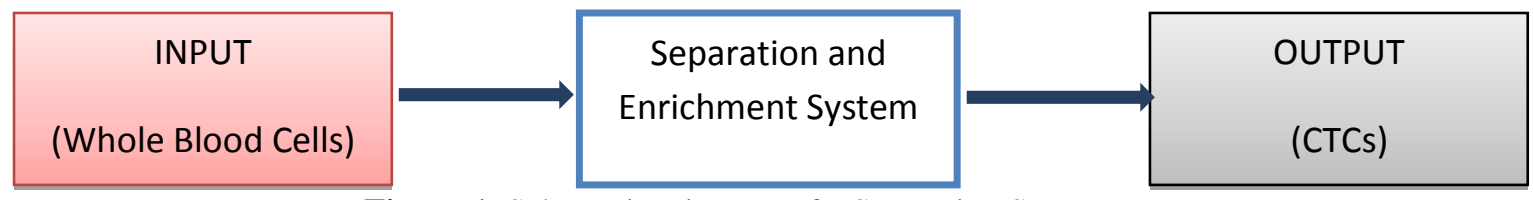

Figure 1. Schematic Diagram of a Separation System

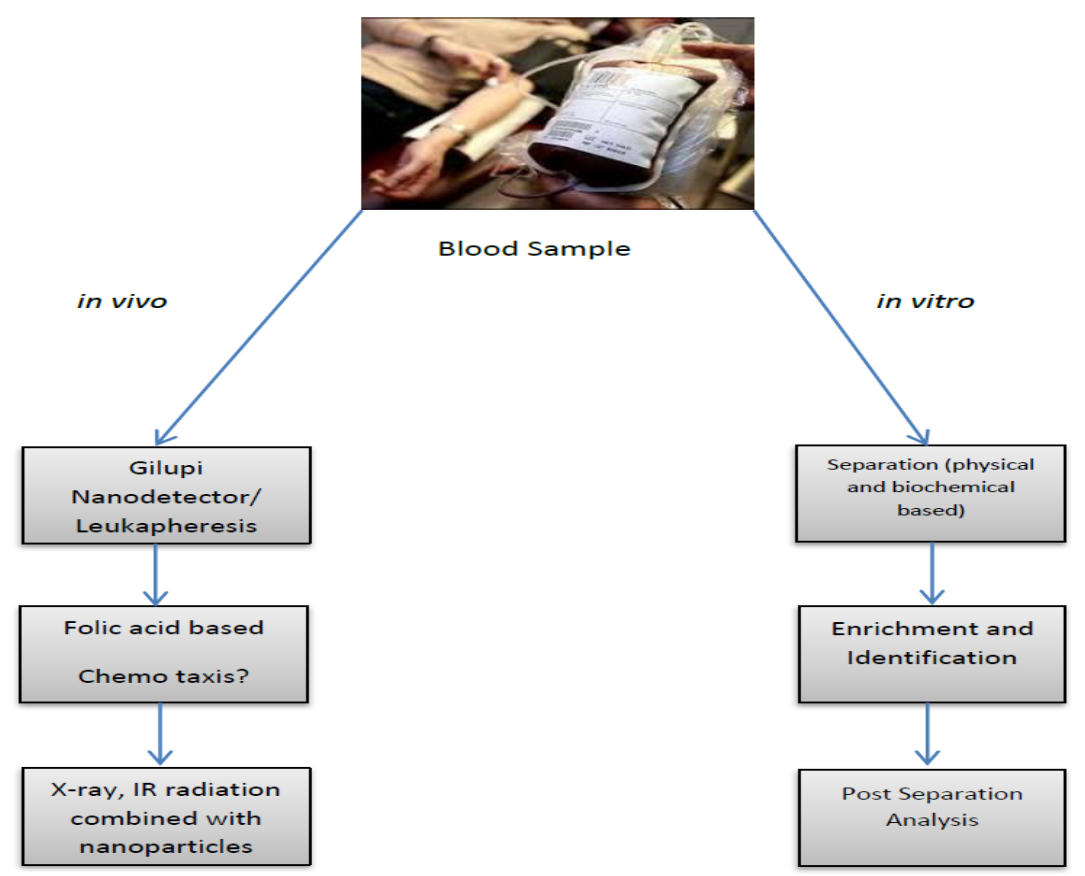

Figure 2.Overview of CTC management 


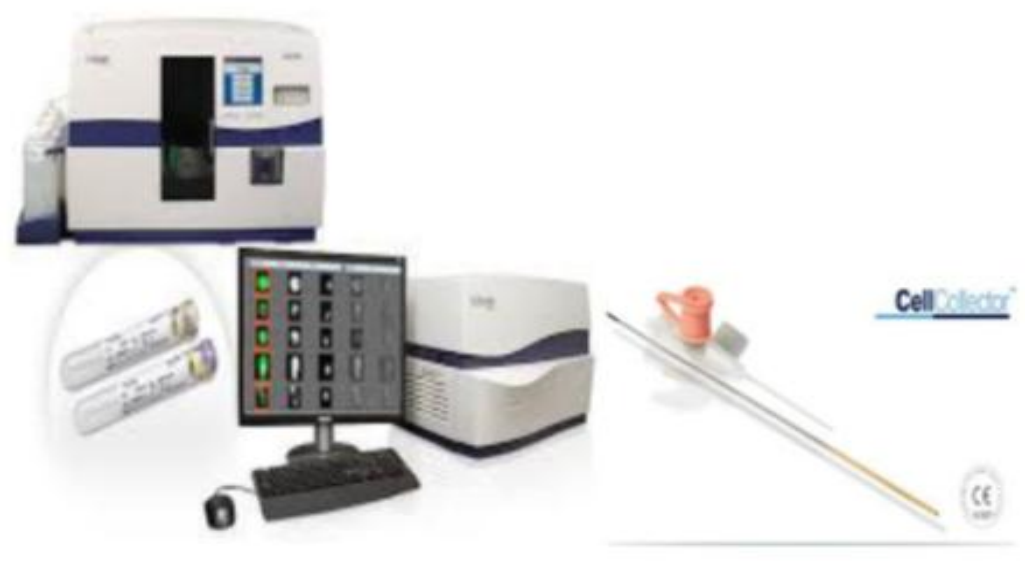

A. CellSystem

B. Gilupi nanodetector

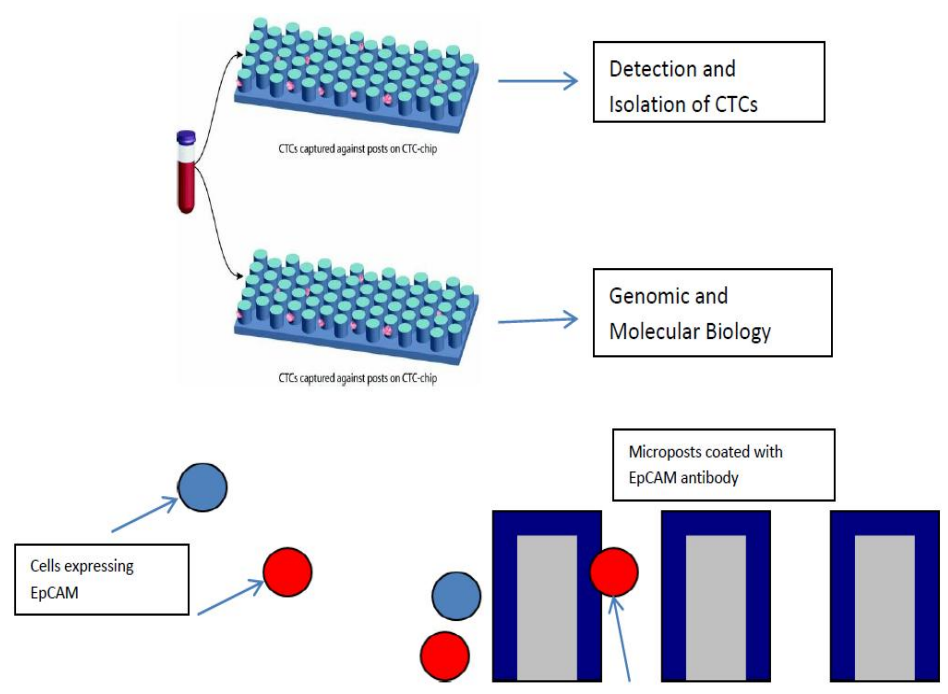

Figure 3. Some CTC separation techniques

Alix-Panabieres, C. and K. Pantel (2014). "Technologies for detection of circulating tumor cells: facts and vision." Lab on a Chip 14(1): 57-62.

Arya, S. K., B. Lim and A. R. A. Rahman (2013). "Enrichment, detection and clinical significance of circulating tumor cells." Lab on a Chip13(11): 1995-2027.

Brandt, B., R. Junker, C. Griwatz, S. Heidl, O. Brinkmann, A. Semjonow, G. Assmann and K. S. Zanker (1996). "Isolation of prostate-derived single cells and cell clusters from human peripheral blood." Cancer Research56(20): 4556-4561.

Castro, C. M., A. A. Ghazani, J. Chung, H. L. Shao, D. Issadore, T. J. Yoon, R. Weissleder and H. Lee (2014). "Miniaturized nuclear magnetic resonance platform for detection and profiling of circulating tumor cells." Lab on a Chip14(1): 14-23.

Cerchia, L. and V. de Franciscis (2010). "Targeting cancer cells with nucleic acid aptamers." Trends Biotechnol28(10): 517525 .

Chambers, A. F., A. C. Groom and I. C. MacDonald (2002). "Dissemination and growth of cancer cells in metastatic sites." Nature Reviews Cancer2(8): 563-572.

Cristofanilli, M., G. T. Budd, M. J. Ellis, A. Stopeck, J. Matera, M. C. Miller, J. M. Reuben, G. V. Doyle, W. J. Allard, L. W. Terstappen and D. F. Hayes (2004). "Circulating tumor cells, disease progression, and survival in metastatic breast cancer." N Engl J Med351(8): 781-791.

Esmaeilsabzali, H., T. V. Beischlag, M. E. Cox, A. M. Parameswaran and E. J. Park (2013). "Detection and isolation of circulating tumor cells: Principles and methods." Biotechnology Advances31(7): 1063-1084.

Fehm, T., A. Sagalowsky, E. Clifford, P. Beitsch, H. Saboorian, D. Euhus, S. D. Meng, L. Morrison, T. Tucker, N. Lane, B. M. Ghadimi, K. Heselmeyer-Haddad, T. Ried, C. Rao and J. Uhr (2002). "Cytogenetic evidence that circulating epithelial cells in patients with carcinoma are malignant." Clinical Cancer Research 8(7): 2073-2084.

Fidler, I. J. (2003). "The pathogenesis of cancer metastasis: the 'seed and soil' hypothesis revisited." Nat Rev Cancer3(6): 453-458.

Gupta, P. B., C. L. Chaffer and R. A. Weinberg (2009). "Cancer stem cells: mirage or reality?" Nat Med15(9): 1010-1012. Hanahan, D. and R. A. Weinberg (2000). "The hallmarks of cancer." Cell100(1): 57-70.

Hayes, D. F. and C. Paoletti (2013). "Circulating tumour cells: insights into tumour heterogeneity." Journal of Internal Medicine274(2): 137-143. 
Hong, B. and Y. L. Zu (2013). "Detecting Circulating Tumor Cells: Current Challenges and New Trends." Theranostics3(6): 377-394.

Hossain, M., Y. Luo, Z. Y. Sun, C. M. Wang, M. H. Zhang, H. Y. Fu, Y. Qiao and M. Su (2012). "X-ray enabled detection and eradication of circulating tumor cells with nanoparticles." Biosensors \& Bioelectronics 38(1): 348-354.

Hou, J. M., M. Krebs, T. Ward, K. Morris, R. Sloane, F. Blackhall and C. Dive (2010). "Circulating tumor cells, enumeration and beyond." Cancers (Basel)2(2): 1236-1250.

Hou, J. M., M. Krebs, T. Ward, R. Sloane, L. Priest, A. Hughes, G. Clack, M. Ranson, F. Blackhall and C. Dive (2011). "Circulating Tumor Cells as a Window on Metastasis Biology in Lung Cancer." American Journal of Pathology 178(3): 989996.

Jain, K. K. (2010). "Advances in the field of nanooncology." Bmc Medicine8.

Jemal, A., F. Bray, M. M. Center, J. Ferlay, E. Ward and D. Forman (2011). "Global Cancer Statistics." Ca-a Cancer Journal for Clinicians61(2): 69-90.

Jin, C., S. M. McFaul, S. P. Duffy, X. Y. Deng, P. Tavassoli, P. C. Black and H. S. Ma (2014). "Technologies for label-free separation of circulating tumor cells: from historical foundations to recent developments." Lab on a Chip 14(1): 32-44.

Kim, G., Y. Yong, H. J. Kang, K. Park, S. I. Kim, M. Lee and N. Huh (2014). "Zwitterionic polymer-coated immunobeads for blood-based cancer diagnostics." Biomaterials35(1): 294-303.

King, J. D., B. P. Casavant and J. M. Lang (2014). "Rapid translation of circulating tumor cell biomarkers into clinical practice: technology development, clinical needs and regulatory requirements." Lab on a Chip 14(1): 24-31.

Lee, H. J., H. Y. Cho, J. H. Oh, K. Namkoong, J. G. Lee, J. M. Park, S. S. Lee, N. Huh and J. W. Choi (2013).

"Simultaneous capture and in situ analysis of circulating tumor cells using multiple hybrid nanoparticles." Biosensors \& Bioelectronics 47: 508-514.

Lu, Y. T., L. B. Zhao, Q. L. Shen, M. A. Garcia, D. X. Wu, S. Hou, M. Song, X. C. Xu, W. H. OuYang, W. W. L. OuYang, J. Lichterman, Z. Luo, X. Xuan, J. T. Huang, L. W. K. Chung, M. Rettig, H. R. Tseng, C. Shao and E. M. Posadas (2013). "NanoVelcro Chip for CTC enumeration in prostate cancer patients." Methods64(2): 144-152.

Molnar, B., A. Ladanyi, L. Tanko, L. Sreter and Z. Tulassay (2001). "Circulating tumor cell clusters in the peripheral blood of colorectal cancer patients." Clinical Cancer Research7(12): 4080-4085.

Nedosekin, D. A., M. A. Juratli, M. Sarimollaoglu, C. L. Moore, N. J. Rusch, M. S. Smeltzer, V. P. Zharov and E. I. Galanzha (2013). "Photoacoustic and photothermal detection of circulating tumor cells, bacteria and nanoparticles in cerebrospinal fluid in vivo and ex vivo." Journal of Biophotonics6(6-7): 523-533.

Paterlini-Brechot, P. and N. L. Benali (2007). "Circulating tumor cells (CTC) detection: Clinical impact and future directions." Cancer Letters 253(2): 180-204.

Phillips, J. A., Y. Xu, Z. Xia, Z. H. Fan and W. H. Tan (2009). "Enrichment of Cancer Cells Using Aptamers Immobilized on a Microfluidic Channel." Analytical Chemistry81(3): 1033-1039.

Stott, S. L., C. H. Hsu, D. I. Tsukrov, M. Yu, D. T. Miyamoto, B. A. Waltman, S. M. Rothenberg, A. M. Shah, M. E. Smas, G. K. Korir, F. P. Floyd, A. J. Gilman, J. B. Lord, D. Winokur, S. Springer, D. Irimia, S. Nagrath, L. V. Sequist, R. J. Lee, K. J. Isselbacher, S. Maheswaran, D. A. Haber and M. Toner (2010). "Isolation of circulating tumor cells using a microvortex-generating herringbone-chip." Proceedings of the National Academy of Sciences of the United States of America107(43): 18392-18397.

Varani, J. (1982). "Chemotaxis of metastatic tumor cells." Cancer Metastasis Rev1(1): 17-28.

Vona, G., L. Estepa, C. Beroud, D. Damotte, F. Capron, B. Nalpas, A. Mineur, D. Franco, B. Lacour, S. Pol, C. Brechot and P. Paterlini-Brechot (2004). "Impact of cytomorphological detection of circulating tumor cells in patients with liver cancer." Hepatology39(3): 792-797.

Witzig, T. E., B. Bossy, T. Kimlinger, P. C. Roche, J. N. Ingle, C. Grant, J. Donohue, V. J. Suman, D. Harrington, J. TorreBueno and K. D. Bauer (2002). "Detection of circulating cytokeratin-positive cells in the blood of breast cancer patients using immunomagnetic enrichment and digital microscopy." Clinical Cancer Research8(5): 1085-1091.

Xu, Y., J. A. Phillips, J. L. Yan, Q. G. Li, Z. H. Fan and W. H. Tan (2009). "Aptamer-Based Microfluidic Device for Enrichment, Sorting, and Detection of Multiple Cancer Cells." Analytical Chemistry81(17): 7436-7442.

Yu, L., S. R. Ng, Y. Xu, H. Dong, Y. J. Wang and C. M. Li (2013). "Advances of lab-on-a-chip in isolation, detection and post-processing of circulating tumour cells." Lab on a Chip13(16): 3163-3182.

Zhang, H., X. Fu, J. Y. Hu and Z. J. Zhu (2013). "Microfluidic bead-based multienzyme-nanoparticle amplification for detection of circulating tumor cells in the blood using quantum dots labels." Analytica Chimica Acta779: 64-71. 